\title{
Nature of Water's Second Glass Transition Elucidated by Doping and Isotope Substitution Experiments
}

\author{
Violeta Fuentes-Landete, ${ }^{1}$ Lucie J. Plaga, ${ }^{2}$ Markus Keppler, ${ }^{2}$ Roland Böhmer, ${ }^{2}$ and Thomas Loerting ${ }^{1, *}$ \\ ${ }^{1}$ Institute of Physical Chemistry, University of Innsbruck, A-6020 Innsbruck, Austria \\ ${ }^{2}$ Fakultät Physik, Technische Universität Dortmund, D-44221 Dortmund, Germany
}

(Received 3 September 2018; revised manuscript received 13 November 2018; published 25 January 2019)

\begin{abstract}
Based on calorimetry and dielectric spectroscopy, the influence of dopants as well as H/D-isotope substitution on the dynamics and thermodynamics of expanded high-density amorphous ice (eHDA) is studied. We find that dopants do not significantly alter the phase behavior, the dielectric relaxation times, and the calorimetric glass transition of eHDA. These observations starkly contrast those made for crystalline ices such as ice $I_{h}$, ice V, ice VI, and ice XII, where suitable dopants enhance the dielectric dynamics by several orders of magnitude and can trigger hydrogen order-disorder transitions, then taking place below the orientational glass transition temperature of undoped samples. This conspicuous contrast to the behavior of crystalline ices strongly argues against point-defect dynamics in amorphous ices and against a previously suggested "crystallinelike" nature of the amorphous ices. Furthermore, H/D substitution also does not affect the calorimetric glass transition in eHDA much, whereas for crystalline ices, the heat capacity increase at the glass transition is roughly halved. In addition, the H/D-isotope shift of the glass transition onset is much larger for crystalline ices than it is for amorphous ices. This observation favors the notion of eHDA's glass transition as a glass-to-liquid transition and is evidence against a mere molecular-reorientation unfreezing at water's second glass transition. Comparing the isotope effect on activation energies for dielectric relaxation with ice $\mathrm{V}$ suggests that in amorphous ice water molecules move translationally above $T_{g}$. Thus, the present work strongly supports that above this glass transition, water does indeed exist in its contested high-density liquid state.
\end{abstract}

DOI: 10.1103/PhysRevX.9.011015

\section{INTRODUCTION}

\section{A. The glass transition in undoped high-density amorphous ice}

Amorphous solids may be observed in more than just a single metastable "phase." In analogy with the polymorphism of crystals, this behavior has been named "amorphous polymorphism" (polyamorphism). Water was the first example for which polyamorphism was discovered by Mishima et al. [1]. Owing to their metastable nature, amorphous ices do not appear in the equilibrium $\mathrm{H}_{2} \mathrm{O}$ phase diagram. However, various amorphous ices of different densities can be prepared in the laboratory [1-8]. High-density amorphous ice (HDA) is prepared at high pressure ( $>1 \mathrm{GPa})$ and low temperature (typically

\footnotetext{
*thomas.loerting@uibk.ac.at
}

Published by the American Physical Society under the terms of the Creative Commons Attribution 4.0 International license. Further distribution of this work must maintain attribution to the author(s) and the published article's title, journal citation, and DOI.
Subject Areas: Chemical Physics, Materials Science, Soft Matter
$77 \mathrm{~K})$ by pressure-induced amorphization of hexagonal ice [2]. At ambient pressure, low-density amorphous ice (LDA) is thermodynamically more stable than HDA [1,9]. Nevertheless, HDA can be quench recovered to ambient pressure. HDA remains kinetically stable at 1 bar for long times, provided the temperature is low enough, whereas unannealed HDA (UHDA) remains stable only up to $100 \mathrm{~K}$, and expanded HDA (eHDA) is stable up to much higher temperatures of $136 \mathrm{~K}[10,11]$. Work on HDA, including calorimetric studies [12], was traditionally carried out with uHDA samples. Only lately has the study of the annealed and expanded forms begun [10,13]. The higher thermal stability in eHDA has opened the door to the discovery of water's second glass transition [14]. For a heating rate of $10 \mathrm{~K} \mathrm{~min}^{-1}$, the onset of water's second glass transition was determined to be $116 \mathrm{~K}$ [14]. In pure water, the calorimetric end point of its second glass transition is hard to access because the polyamorphic transition intervenes [14]. A recent study by Ruiz et al. [15] shows, however, that the presence of $\mathrm{LiCl}$ allows for the observation of the end point before the polyamorphic transition commences. The nature of eHDA above $T_{g}$ is 
still unclear and contested, so we focus here on resolving the question on the molecular motions taking place at water's recently discovered second glass transition, especially on the question of whether or not a liquid state is attained.

\section{B. Glass and hydrogen-ordering transitions in crystalline ices}

The electrical properties of ice are very sensitive to small concentrations of certain impurities that can be incorporated in the hydrogen-bonded network to generate point defects, specifically ionic defects and/or Bjerrum defects [16]. Examples of dopants that are soluble up to a few parts per million are $\mathrm{HF}, \mathrm{HBr}, \mathrm{HCl}, \mathrm{KOH}$, and $\mathrm{NH}_{3}$. These molecules are incorporated in the crystal lattice by substitution of a water molecule [17]. In the past, such dopants were used to influence the hydrogen-bond dynamics in crystalline ices [19-23]. Dopants have allowed one to unlock kinetically hindered but thermodynamically favored transitions to hydrogen-ordered ices [16]. The role of these dopants is to enhance microscopic dynamics [18,24,25]. This enhancement is key to inducing hydrogen-ordering transitions at $T<150 \mathrm{~K}$, where otherwise molecular motion is too slow for ordering processes to take place on typical laboratory timescales. However, it is still unclear why some dopants enhance the dynamics in crystalline ices whereas others do not [26,27].

For illustrative purposes, Fig. 1 shows calorimetric experiments comparing undoped, unsuitably, and suitably

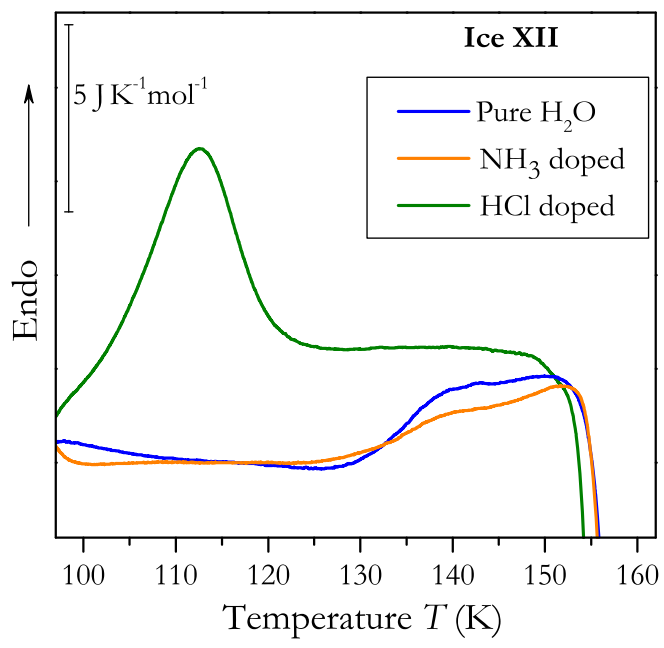

FIG. 1. DSC heating scans recorded at $30 \mathrm{~K} \mathrm{~min}^{-1}$ and ambient pressure for doped and undoped ice XII. The signal is normalized to 1 mol. Data taken from Fig. 1 of Köster et al. [25]. The endothermic peak observed for the $\mathrm{HCl}$-doped sample (green trace) represents the transition from hydrogen-ordered ice XIV to hydrogen-disordered ice XII. For the pure water sample (blue trace) and the $\mathrm{NH}_{3}$-doped sample (orange trace), the glass transition onset near $130 \mathrm{~K}$ indicates that atomic mobility is thawed above this temperature. doped ice XII samples. For undoped ice XII, one observes the (un)locking of the $\mathrm{H}$-atom motion as a glass transition at $129 \mathrm{~K}$ (see Fig. 1, blue trace). At $T<129 \mathrm{~K}, \mathrm{H}$-atom motion is frozen, so the isobaric heat capacity $c_{p}$ is small. At $T>129 \mathrm{~K}, \mathrm{H}$-atom motion is thawed, giving rise to a significantly higher $c_{p}$ in the mobile state. Qualitatively, the same picture is observed for $\mathrm{NH}_{3}$-doped ice XII. Its calorimetric trace (Fig. 1, orange trace) is very similar to the one for the undoped sample, which demonstrates that $\mathrm{NH}_{3}$ is a dopant that is unsuitable to enhance the $\mathrm{H}$-atom dynamics sufficiently to trigger a $\mathrm{H}$-ordering transition. By contrast, $\mathrm{HCl}$ doping is found to be suitable: An endothermic peak is observed upon heating (Fig. 1, green trace) rather than a glass transition. The endotherm allows one to access the latent heat taken up at the disordering transition occurring near $102 \mathrm{~K}$. This observation reveals that near $102 \mathrm{~K}$, the $\mathrm{H}$-atom dynamics is still sufficiently fast to achieve, upon cooling, the entropically favored ordered state, ice XIV [22]. Quantitatively, an enhancement of the dielectric relaxation by up to 5 orders of magnitude was measured for $\mathrm{HCl}$-doped ice XII samples when compared to $\mathrm{NH}_{3}$-doped or undoped samples [25]. That is, by enhancing the hydrogen dynamics through suitable extrinsic point defects, it is possible to unlock a transition to a hydrogen-ordered phase. Similar results were reported for other disordered ice phases, e.g., ice V or ice VI [28,29]. For all high-pressure ice phases, $\mathrm{HCl}$ was found to be the most efficient dopant, whereas for the low-pressure hexagonal ice, $\mathrm{KOH}$ was found to be most efficient [19].

\section{Doped and H/D-substituted eHDA}

A number of studies emphasized that a high similarity between amorphous ices and crystalline ices might exist [30-32]. Furthermore, a glass transition akin to the ones shown in Fig. 1 was observed for eHDA [14]. It has been debated whether at the glass transition of amorphous ices only reorientations unfreeze or whether additionally also translational motion of $\mathrm{H}_{2} \mathrm{O}$ molecules is unlocked $[14,33,34]$. That is, it is of interest to find out whether oxygen atoms remain immobile and only hydrogen atoms move around them, or whether instead, the complete water molecule becomes mobile upon heating above the glass transition. In the spirit of the latter scenario, some of us [14] claimed that the glass transition of eHDA "involves liquidlike translational mobility of water molecules." Shephard and Salzmann, on the other hand, argued that the glass transition in eHDA is an orientational glass transition, just like the one occurring in undoped ice VI [33] or ice XII [35]. Recently, this controversy was summarized by stating that "at the orientational glass transition of ice XII the oxygen atoms do remain at their lattice positions, but this may or may not be so at the glass transition of amorphous ices" [36].

For amorphous ices, a local $\mathrm{H}$ ordering can build up and persist only if the glass transition is governed by 
point-defect mobility, which leaves the O-atom topology unaffected. This scenario was suggested by Fisher and Devlin [34], who considered that suitable doping of amorphous ices should speed up the dynamics of Bjerrum and ionic point defects with respect to the pure sample. One may speculate that such a dynamic enhancement could then give rise to some kind of local $\mathrm{H}$ ordering in eHDA. If, however, the $\mathrm{O}$ atom's topology changes on the timescale of the experiment, then any local $\mathrm{H}$ ordering would be only transient. Transient $\mathrm{H}$ order would be depleted above $T_{g}$ once the $\mathrm{O}$ atoms have moved several $\mathrm{O}-\mathrm{O}$ distances. Consequently, in this scenario, extrinsic point defects introduced through dopants should not have a sizeable influence on the dynamics. Reasons for a lack of significant doping effects might be the nature of the amorphous structure, which allows for an intrinsic violation of the ice rules and/or a depletion of dopants from the bulk [37].

Previously, only a limited number of studies addressed the effects of dopants on amorphous ices with the result that doping with $\mathrm{KOH}, \mathrm{HF}, \mathrm{NH}_{3}$, or $\mathrm{NH}_{4} \mathrm{~F}$ does not enhance the dynamics in HDA $[37,38]$. It is the purpose of the present study not only to examine the effect of dopants on eHDA more comprehensively but also to exploit the much enhanced experimentally accessible temperature range that was opened up by the advent of eHDA in order to gain new insights into the nature of its glass transition. Particularly, we take $\mathrm{HCl}$ doping into consideration. Based on its propensity to induce order in crystalline polymorphs that are similar to eHDA in terms of local structural order, $\mathrm{HCl}$ would appear as the most promising candidate: An important question is whether or not, analogous to crystalline ices (see Fig. 1), a local variant of a $\mathrm{H}$ ordering or disordering can be achieved for suitably doped amorphous ices.

Isotope substitution experiments can be helpful to explore whether-above the glass transition temperature $T_{g}$ of amorphous ice-hydrogen and oxygen atoms or just the hydrogen atoms are mobile. To this end, we compare isotope effects at the glass transitions of amorphous and crystalline ices with the goal to aid in resolving the controversy of the mechanisms governing the glass transitions of amorphous ices. In particular, we look at changes of the dynamics of the heat capacity step $\Delta c_{p}$ and of shifts in $T_{g}$ to check whether or not any such changes are similar to those of crystalline ices.

\section{EXPERIMENT}

We study eHDA samples rather than uHDA, since the latter has a much lower thermal stability and does not show the second glass transition prior to the polyamorphic transition. Undoped and doped $\mathrm{H}_{2} \mathrm{O}\left(\mathrm{D}_{2} \mathrm{O}\right)$ eHDA samples are prepared from pure (heavy) water or solutions containing $0.01-\mathrm{M} \mathrm{HCl}(\mathrm{DCl}), \mathrm{HBr}, \mathrm{HF}, \mathrm{NH}_{3}$, and $\mathrm{KOH}$. The sample preparation route follows our earlier work [13]. In short, $600 \mu \mathrm{l}$ of an aqueous solution is pipetted into a
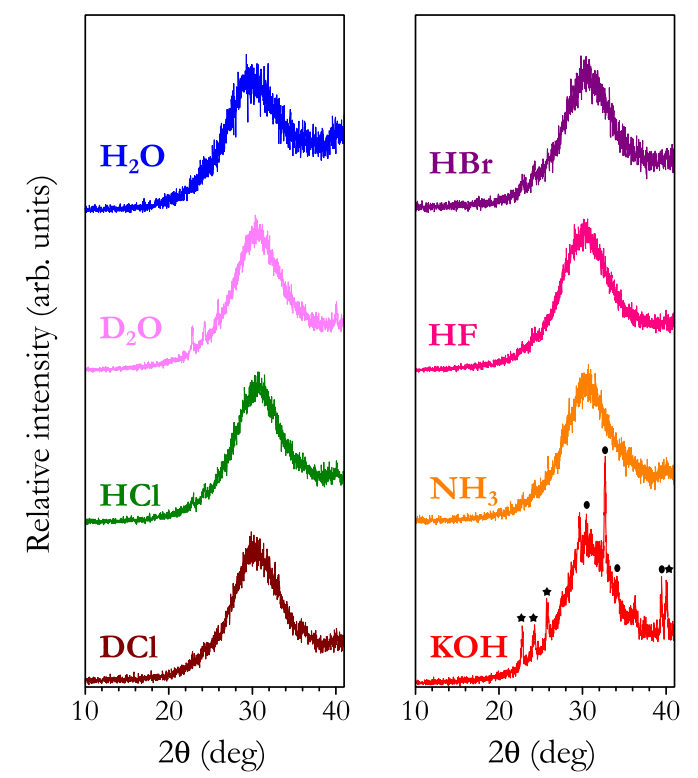

FIG. 2. Powder x-ray characterization of eHDA samples (recorded at $80 \mathrm{~K}$ ) in undoped form or with the indicated dopants. Stars and circles mark hexagonal ice and ice XII, respectively.

piston cylinder apparatus of 8-mm bore diameter at $77 \mathrm{~K}$, yielding hexagonal ice. All samples are kept in a container made of indium to avoid sudden pressure drops during compression [2]. Hexagonal ice is then pressurized to $1.8 \mathrm{GPa}$ at $77 \mathrm{~K}$ inducing amorphization of the sample and formation of uHDA [2]. uHDA transforms to eHDA by isobaric annealing at $1.1 \mathrm{GPa}$ and approximately $160 \mathrm{~K}$, cooling to $140 \mathrm{~K}$, and isothermal decompression at a controlled rate of $20 \mathrm{MPa} \mathrm{min}^{-1}$ to $0.1 \mathrm{GPa}$ [39]. For the $\mathrm{D}_{2} \mathrm{O}$ samples, $143 \mathrm{~K}$ is used instead of $140 \mathrm{~K}$ [40]. Subsequently, the samples are quench recovered to ambient pressure and then transferred under liquid nitrogen to the calorimeter or to a Dewar that is shipped to Dortmund where the dielectric measurements are carried out. A description of the procedures relevant for the dielectric experiments can be found elsewhere [14,24].

All samples are characterized using x-ray diffraction. For all doped samples, the diffraction patterns match the ones for eHDA that are reported in the literature [39]; i.e., the halo peak maximum is located at approximately $29.5^{\circ}-30.0^{\circ}\left(\mathrm{Cu}-\mathrm{K}_{\alpha, 1}, \lambda=1.5406 \AA\right)$; see Fig. 2 . The only exception is $\mathrm{KOH}$ doping, which results in a slightly different pattern that contains some sharp Bragg peaks (see Fig. 2 and Sec. III A).

A differential scanning calorimeter (PerkinElmer, DSC 8000 ) is used for the calorimetric analysis. Like in previous work [25,35,41], DSC scans are recorded upon heating the samples at a rate of $50 \mathrm{~K} \mathrm{~min}^{-1}$ from 93 to $253 \mathrm{~K}$ using aluminum crucibles. After heating each sample to $253 \mathrm{~K}$ and subsequent recooling to $93 \mathrm{~K}$, a second heating scan of now ice $I_{h}$ is recorded and subtracted as a baseline from 
the first scan. The mass of the sample cannot be obtained by weighing; therefore, we calculate it from the endothermic melting of ice $I_{h}$ by using the value of $6012 \mathrm{~J} \mathrm{~mol}^{-1}$ $\left(6280 \mathrm{~J} \mathrm{~mol}^{-1}\right)$ as enthalpy of fusion of $\mathrm{H}_{2} \mathrm{O}\left(\mathrm{D}_{2} \mathrm{O}\right)$. Usually, this determination yields sample masses of 8-15 mg. All thermograms are normalized to $1 \mathrm{~mol}$. Every DSC curve shown here is the average of at least three samples and three repetitions for each.

\section{RESULTS AND DISCUSSION}

\section{A. Influence of dopants and isotope substitution on phase behavior}

In order to address the question of whether or not "doping" is effective in altering the dynamic and thermodynamic properties of amorphous ice, we prepare doped eHDA samples and first analyze them by DSC. The thermograms obtained for pure $\mathrm{H}_{2} \mathrm{O}$ and $\mathrm{D}_{2} \mathrm{O}$ eHDA samples are shown in Fig. 3 (top two traces, left panel) and serve as reference. The remaining traces in Fig. 3 represent an overview of DSC scans for ices containing the indicated dopants. All samples show two exotherms in the range of $120-200 \mathrm{~K}$ at very similar temperatures. These features reflect the thermal events related to the amorphous-amorphous transition, that is, from eHDA to LDA. For the large heating rate used in this work, this transition

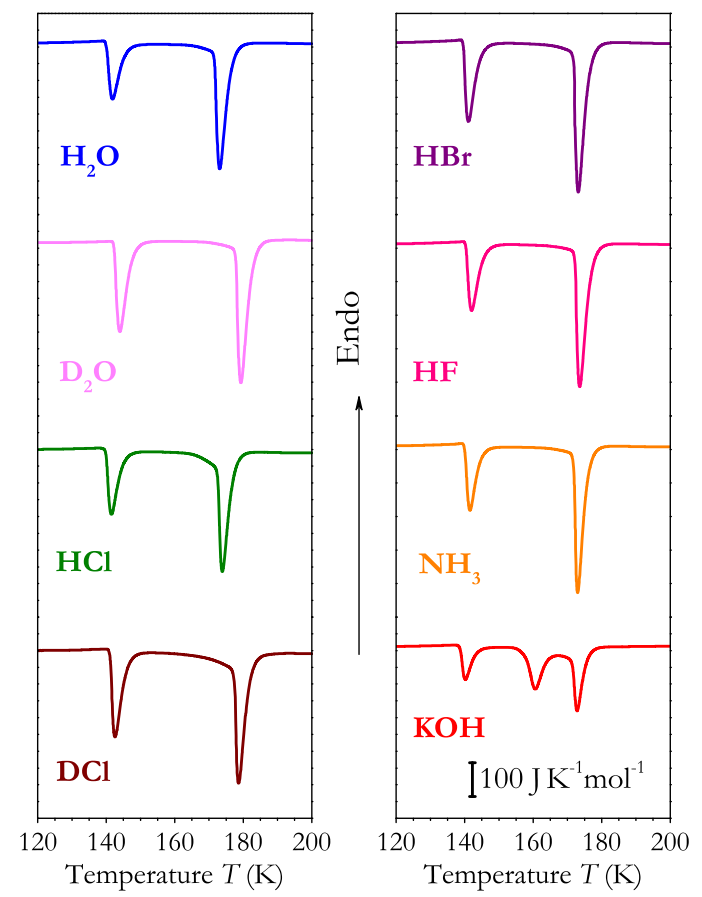

FIG. 3. Calorimetric scans obtained for quench-recovered eHDA samples. The samples are heated in the DSC at ambient pressure with $50 \mathrm{~K} \mathrm{~min}^{-1}$. Curves are shifted vertically for clarity. All curves are shown after subtraction of the second heating scan (this scan on ice $I_{h}$ serves as a baseline). The different colors refer to different dopants or isotopes as indicated. appears near $140 \mathrm{~K}$, and the crystallization from LDA to ice $I_{c}$ here shows up near $180 \mathrm{~K}$. That is, doping does not affect the phase transition behavior of eHDA, with the exception of the KOH-doped sample (cf. Fig. 3, right panel, red bottom trace). This scan shows a third exotherm which exhibits its minimum at a temperature of approximately $160 \mathrm{~K}$ indicating the ice XII to ice $I_{c}$ transition. The preparation protocol results in a mixture of ice XII and eHDA rather than pure eHDA. This finding is confirmed by $\mathrm{X}$-ray diffraction showing ice XII Bragg peaks on top of the eHDA halo peak (marked by circles in Fig. 2). This result will be scrutinized in more detail in future work.

Figure 4 shows an evaluation of the temperature of transformation from eHDA to LDA [Fig. 4(a)] and the crystallization temperature from LDA to ice $I_{c}$ [Fig. 4(b)] for all dopants studied. These plots reveal that, independent of the dopant, a very similar behavior is detected. The onset temperature of the polyamorphic transition is $139 \pm 1 \mathrm{~K}$, and the minimum temperature of the crystallization is $174 \pm 1 \mathrm{~K}$, consistent for all $\mathrm{H}_{2} \mathrm{O}$-based samples [42]. Thus, none of the dopants is found to alter the transformation

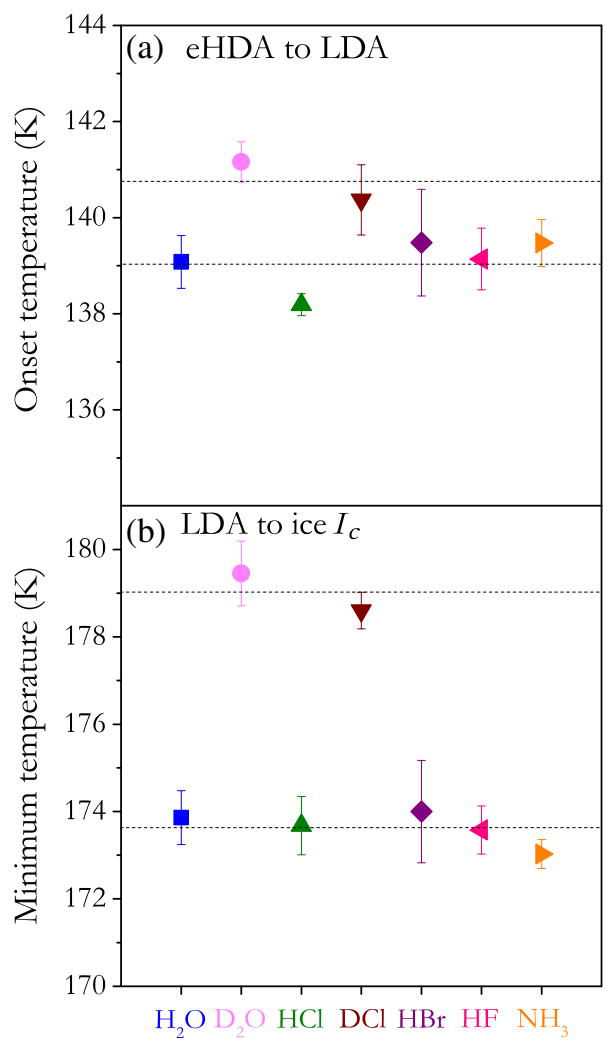

FIG. 4. (a) Onset temperature of the transformation from eHDA to LDA for different isotopes or dopants. (b) Minimum peak temperature for the transition from LDA to ice $I_{c}$ for different isotopes or dopants. Black dashed horizontal lines describe the average values for the $\mathrm{H}_{2} \mathrm{O}$ and/or $\mathrm{D}_{2} \mathrm{O}$ samples. The error bars reflect the standard deviations obtained from repeated measurements. The colors are consistent with the ones used in Fig. 3. 
temperature. A difference in transformation temperatures is noted merely for samples containing $\mathrm{D}_{2} \mathrm{O}$ instead of $\mathrm{H}_{2} \mathrm{O}$. The isotope effect on the polyamorphic transition amounts to approximately 2 K [Fig. 4(a)], whereas the crystallization temperature displays a remarkable shift of approximately 5-6 K [Fig. 4(b)]. The crystallization shows a slightly larger isotope effect than the melting transition of hexagonal ice where H/D substitution leads to a change of $3.8 \mathrm{~K}$ [43]. That is, except for the $\mathrm{KOH}$-doped sample, which is not included in Fig. 4, the phase transformation kinetics is not at all affected by the dopants. Both the polyamorphic transition and the crystallization involve a rearrangement of the O-atom network. Hence, our statement is analogous to saying that the O-atom dynamics is not affected by the studied dopants.

\section{B. Influence of dopants and isotopes on the glass transition}

\section{Dielectric studies}

In order to assess the $\mathrm{H}$-atom dynamics, we also carry out dielectric spectroscopy studies of variously doped amorphous ices. In Fig. 5(a), we show the dielectric loss of $\mathrm{NH}_{3}$-doped eHDA for temperatures ranging from 108 to $126 \mathrm{~K}$. As the temperature is increased, the dielectric loss peaks shift to higher frequencies. Then, at $129 \mathrm{~K}$ the peaks shift back to lower frequencies; see the crosses in Fig. 5(a). Similar to the observations made for undoped samples [14], this indicates that the transition to LDA takes place between 126 and $129 \mathrm{~K}$. The polyamorphic

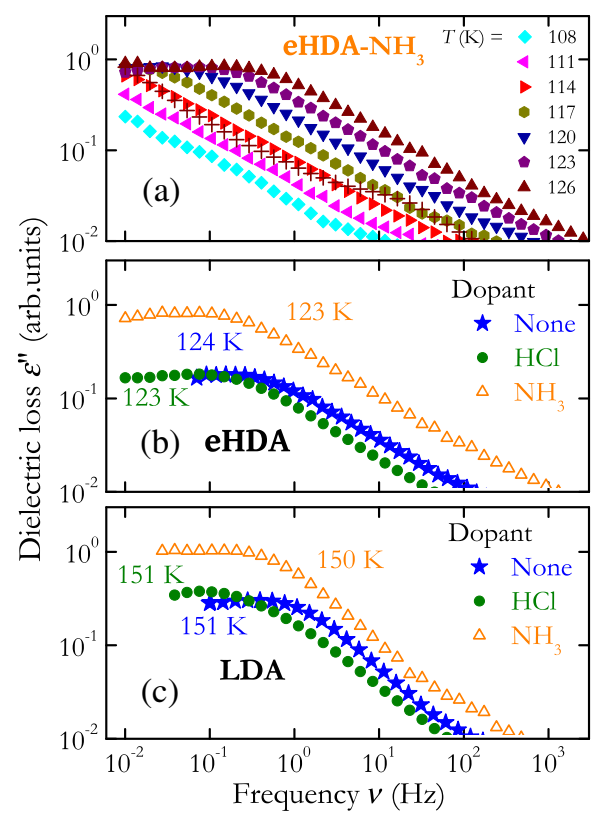

FIG. 5. (a) Frequency-dependent dielectric loss spectra of $\mathrm{NH}_{3}$-doped eHDA. For comparison, the crosses indicate the dielectric loss spectrum obtained at $129 \mathrm{~K}$. It refers to $\mathrm{NH}_{3}$-doped LDA. Frame (b) shows loss spectra for undoped, HCl-doped, and $\mathrm{NH}_{3}$-doped eHDA. Frame (c) presents corresponding data for doped and undoped LDA. transition occurs approximately $10 \mathrm{~K}$ lower here than indicated in Fig. 4(a) because of the much slower heating employed for dielectric studies.

Figures 5(b) and 5(c) compare the dielectric losses of $\mathrm{NH}_{3}$-doped eHDA and LDA with those of undoped [14] as well as with $\mathrm{HCl}$-doped samples. One recognizes that doping has only a minor impact on the dynamics of these ice forms. In particular, it is clear that the $\mathrm{HCl}$ and $\mathrm{NH}_{3}$ dopants do not lead to a dynamical enhancement. This finding is in accord with high-pressure dielectric studies of another KOH-doped amorphous high-density ice [37]. However, the absence of a pronounced sensitivity to doping is in stark contrast to the observations made for numerous crystalline ices where doping using $\mathrm{HCl}, \mathrm{HF}, \mathrm{KOH}$, or other suitable agents typically leads to significant dynamical enhancements of up to several decades $[18,24,25,44]$.

Temperature-dependent dipolar relaxation times $\tau=$ $\left(2 \pi \nu_{\max }\right)^{-1}$ are obtained directly from the peak frequencies $\nu_{\max }$ evident from Fig. 5(a) for a $\mathrm{NH}_{3}$-doped sample. The filled symbols in Fig. 6(a) refer to that sample and the half-filled symbols to the peak frequencies of a second $\mathrm{NH}_{3}$-doped sample. However, at low temperatures the peaks move out of the experimental frequency window. Then, as exploited in several previous studies on ices, we apply frequency-temperature superposition [24]. Explicit illustrations of this kind of data analysis can be found, e.g., in Refs. $[9,45]$. The time constants assessed using that procedure are represented in Fig. 6(b) as open symbols (or as dotted symbols for the second sample). Apart from the relaxation times thus estimated for $\mathrm{NH}_{3}$-doped eHDA and LDA, Fig. 6 also illustrates the temperature protocol employed for these measurements. The relaxation times are presented in a way that renders it obvious that they approximately follow an Arrhenius law $\tau=\tau_{0} \exp (E / R T)$, where $\tau_{0}$ denotes a preexponential factor and $E$ an activation energy.

To place the temperature-dependent relaxation times of $\mathrm{NH}_{3}$-doped eHDA and LDA into a broader context, in Fig. 6(b) we compare them with those for undoped [14] and $\mathrm{HCl}$-doped (hydrogenated) samples. Data for deuterated amorphous ices are included as well $[46,47]$. One recognizes that the effective activation energies for all of the differently doped eHDA ices are close to each other; the same holds for the LDA samples. In particular, while substitution of hydrogens by deuterons slows the dynamics significantly [46,47], Fig. 6(b) confirms the absence of any doping-induced dynamical enhancement in eHDA as well as in LDA. Thus, while it is conceivable that-at least in the by and large locally tetrahedrally coordinated LDA building blocks [48,49]—an amorphous network exists on which the Bernal-Fowler rules could be definable, a doping-induced violation of ice rules is not evident from the current experiments. In eHDA, the water molecules are roughly fivefold coordinated [50]. The corresponding high-density network is thus much more defective already 
Temperature $T(\mathrm{~K})$

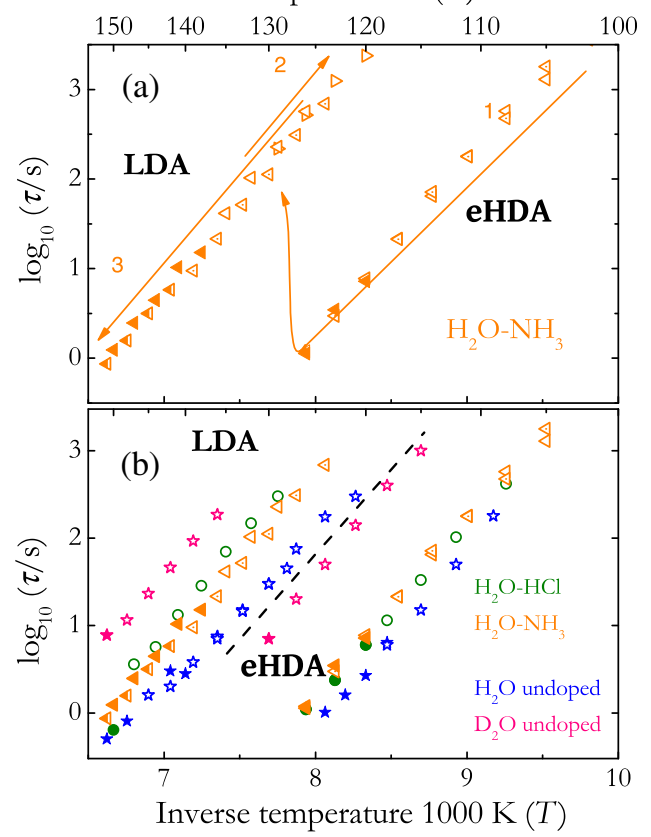

FIG. 6. (a) Dielectric relaxation times estimated for a $\mathrm{NH}_{3}$-doped eHDA sample. The numbered arrows indicate the applied temperature protocol. Triangles pointing to the left refer to data obtained during heating, those pointing to the right upon cooling. Filled and half-filled symbols refer to relaxation times assessed directly from the peak frequencies for two different samples; the open and dotted symbols are obtained by means of frequency-temperature superposition. (b) Compilation of relaxation times for various doped and undoped eHDA and LDA samples as obtained in heating runs. Relaxation times to the left of the dashed line refer to LDA, those to the right to eHDA. Data for undoped (hydrogenated as well as deuterated) samples are from previous work [14,46,47]. These latter data indicate that the dynamics in deuterated LDA (eHDA) is of the order of 20 (50) times slower as compared to that in hydrogenated LDA (eHDA).

intrinsically and, consequently, likely to be far less susceptible to defect doping than the low-density network is. It is also remarkable that in several high-pressure crystalline ices, $\mathrm{NH}_{3}$ doping generates anomalously low energy barriers [24,25], while for the amorphous ices from Fig. 6(b), "normal" energy barriers are observed for this dopant.

All these observations demonstrate that the dopingfacilitated dynamics in the presently studied amorphous ices are different from those in the crystalline phases. The latter ices are governed by local hydrogen moves on a tetrahedral water network in which nonvibrational oxygen motion apparently does not play any role. Of course, it cannot be ruled out that the dopants used in this work are just unsuitable to trigger a dynamical enhancement. However, in view of the fact that (in crystalline ices) the currently employed dopants can create all sorts and combinations of ionic and Bjerrum defects, this option appears highly unlikely.

\section{Differential scanning calorimetry}

Let us now turn to the low-temperature dynamics in eHDA taking place prior to the polyamorphic transition, as assessed by DSC. Figure 7 represents a magnification of Fig. 3 in the range of $95-145 \mathrm{~K}$. It is immediately evident that an endotherm similar to the one in Fig. 1 for suitably doped crystalline ice (green line) is not observed. Instead, all scans reveal a glass transition similar to the ones in Fig. 1 for undoped or unsuitably doped ice. That is, a hydrogen-ordering transition for eHDA is not observed in our study. The eHDA-LDA transition seen near $140 \mathrm{~K}$ is preceded by a glass transition, for a heating rate of $50 \mathrm{~K} \mathrm{~min}^{-1}$ located at an onset temperature of $125.0 \pm$ $0.9 \mathrm{~K}$ (as determined using the tangent intersection method; see Fig. 7, blue trace from undoped eHDA). For a heating rate of $10 \mathrm{~K} \mathrm{~min}^{-1}$ [14], Amann-Winkel et al. located the glass transition at $116 \pm 2 \mathrm{~K}$. The difference of $9 \mathrm{~K}$ arises from the fivefold increase in the heating rate and contains two contributions: approximately $2.5 \pm 1 \mathrm{~K}$ of thermal lag of the instrument and $6.5 \pm 1 \mathrm{~K}$ of shift caused by the response of the sample itself that at higher rates is probed at higher effective frequencies.

The phenomenology of the eHDA glass transition is evaluated quantitatively in Fig. 8, where we plot the glass transition onset temperature [Fig. 8(a)] and the heat

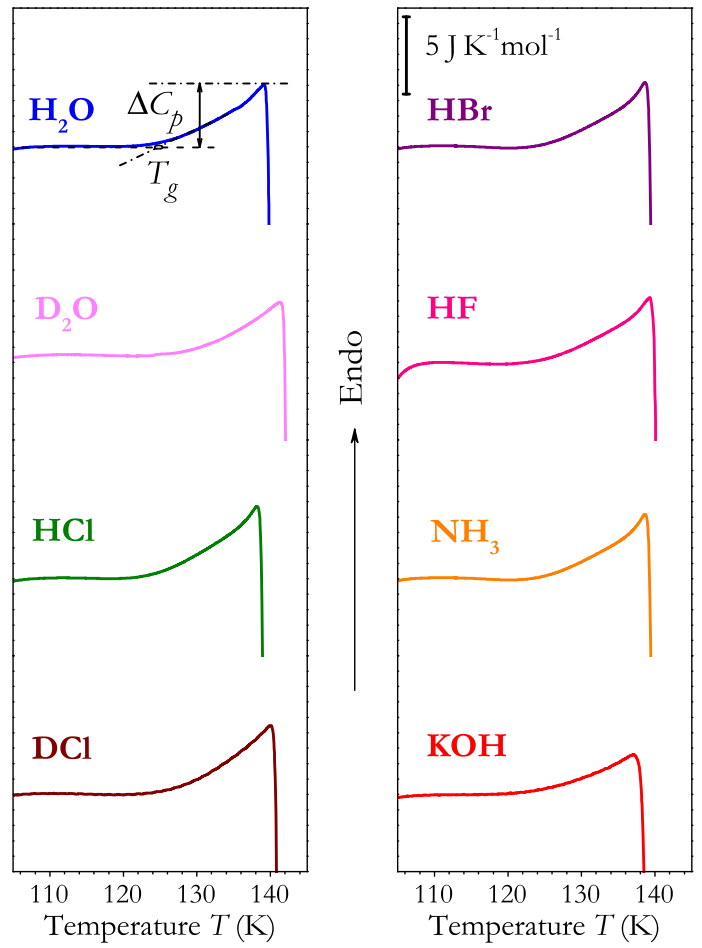

FIG. 7. Calorimetric scans from quench-recovered eHDA samples heated at ambient pressure at a rate of $50 \mathrm{~K} \mathrm{~min}^{-1}$. This figure represents a magnification of the low-temperature part from Fig. 3. A representation of the evaluation method of $\Delta c_{p}$ and $T_{g}$ is shown here by dashed lines. 


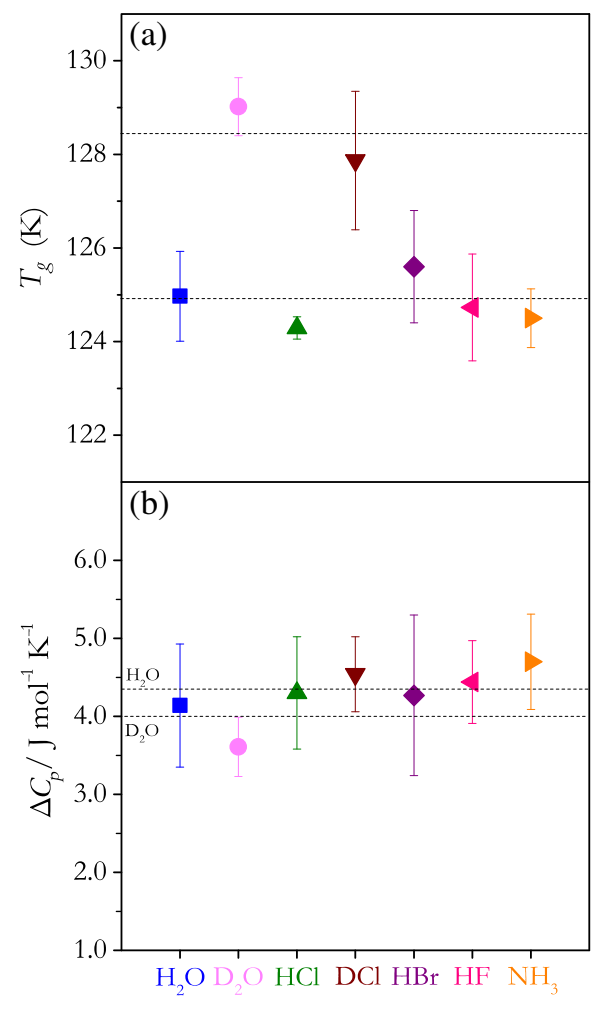

FIG. 8. (a) Onset glass transition temperature $T_{g}$ and (b) heat capacity change $\Delta c_{p}$ for eHDA at $T_{g}$ for different isotopes or dopants. Black dashed horizontal lines describe the average value for the $\mathrm{H}_{2} \mathrm{O}$ and/or $\mathrm{D}_{2} \mathrm{O}$ samples.

capacity change at the glass transition $\Delta c_{p}$ [Fig. 8(b)] for all dopants used. The onset of the glass transition remains the same, namely, $125 \pm 1 \mathrm{~K}$, when using $\mathrm{HCl}-, \mathrm{HBr}-$, HF-, and $\mathrm{NH}_{3}$-doped samples. Only after isotope substitution, we observe a shift to higher temperatures, on average by 3-4 K, for both doped and undoped $\mathrm{D}_{2} \mathrm{O}$ samples. This isotope shift is in agreement with that previously observed by Gainaru et al. (see Fig. 2G in Ref. [46] for rates of $50 \mathrm{~K} \mathrm{~min}^{-1}$ ).

By contrast, the change in heat capacity at the glass transition $\Delta c_{p}$ is similar for all studied dopants and isotopes, considering the error bars given by the reproducibility of the experiment. Specifically, the heat capacity increases by $4.3 \pm 0.8 \mathrm{~J} \mathrm{~K}^{-1} \mathrm{~mol}^{-1}$ for all $\mathrm{H}_{2} \mathrm{O}$-based samples and by $4.0 \pm 0.6 \mathrm{~J} \mathrm{~K}^{-1} \mathrm{~mol}^{-1}$ for the two $\mathrm{D}_{2} \mathrm{O}$ based samples [see the horizontal dashed lines in Fig. 8(b)]. The former value is in agreement with the one published by Amann-Winkel et al. $\left(4.8 \mathrm{~J} \mathrm{~K}^{-1} \mathrm{~mol}^{-1}\right)$ [14]. Please note that Ruiz et al. have observed a spike as part of the glass transition in eHDA [15]. They have suggested the spike to be caused by adiabatic cooling caused by the $25 \%$ expansion at the polyamorphic transition. Without a spike, $\Delta c_{p}$ was given as $2.3 \pm 0.5 \mathrm{~J} \mathrm{~K}^{-1} \mathrm{~mol}^{-1}$. In Fig. 7, we do not observe a spike, so we compare our values with the ones from Amann-Winkel et al. [14].

As the only exception, the $\mathrm{KOH}$-doped sample shows a lower $\Delta c_{p}$ than all the other ices. This finding reflects that only part of that sample is eHDA, and the remaining part is ice XII. Thus, KOH-doping data are not included in Fig. 8.

\section{Isotope effect on $\Delta c_{p}$ and on timescales near the glass transition}

The finding that $\Delta c_{p}$ at the glass transition is barely affected by isotope substitution or by doping is of high interest, especially in the context of the question regarding the kind of mobility that is unlocked at $T_{g}$. Here, we discuss whether at the glass transition of amorphous ices reorientational motion prevails or whether additionally also translational motion of water molecules is taking place. Figure 9 demonstrates the effect of H/D substitution on $\Delta c_{p}$ at the
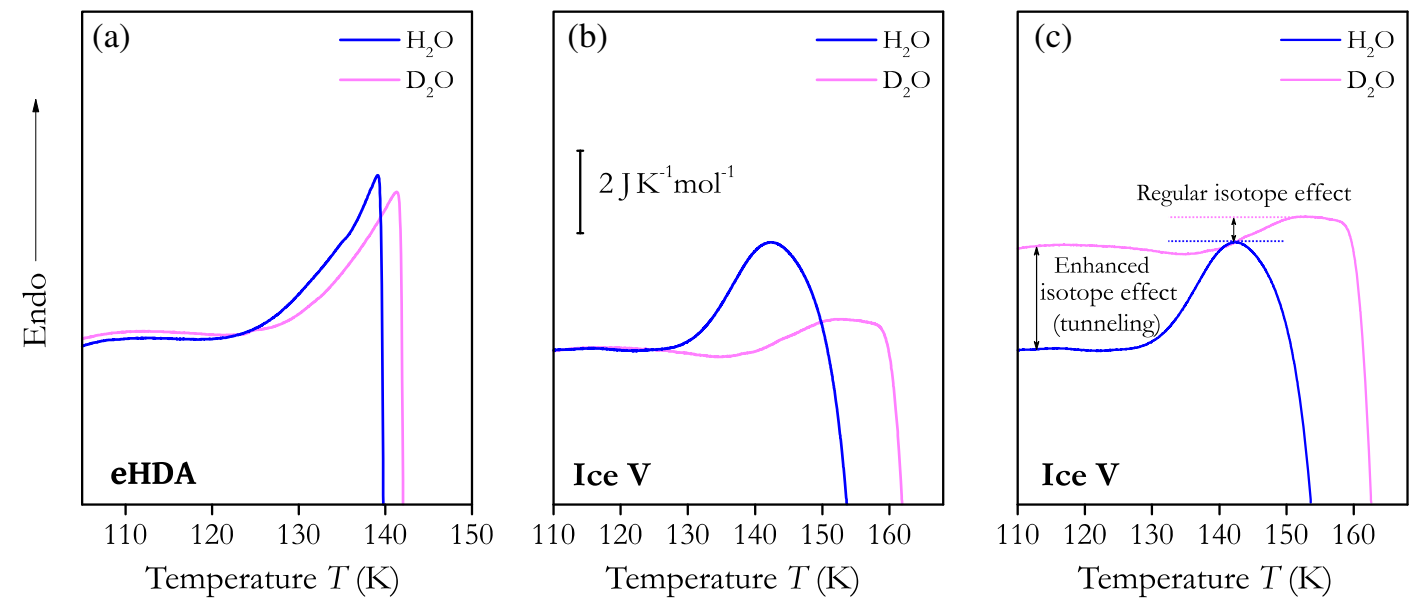

FIG. 9. Isotope effect on the heat flow at the glass transition of (a) eHDA measured at $50 \mathrm{~K} \mathrm{~min}^{-1}$ and (b) ice V at $30 \mathrm{~K}$ min ${ }^{-1}$. Panel (c) suggests that the larger isotope effect for ice $\mathrm{V}$ is caused by strongly enhanced H-quantum tunneling below $T_{g}$. The ice $\mathrm{V}$ samples are prepared following the protocol described by Salzmann et al. [28]. The signal is normalized to 1 mol, and the scans are moved vertically for clarity. 
TABLE I. Onset glass transition temperatures $\left(T_{g}\right)$, difference in $T_{g}$ between $\mathrm{H}_{2} \mathrm{O}$ and $\mathrm{D}_{2} \mathrm{O}\left(\Delta T_{g}\right)$, and change in heat capacity $\left(\Delta c_{p}\right)$ at the glass transition for different crystalline ices for $\mathrm{H}_{2} \mathrm{O}$ and $\mathrm{D}_{2} \mathrm{O}$.

\begin{tabular}{lcccc}
\hline \hline Crystalline ice sample & $\begin{array}{c}\text { Heating rate } \\
\left(\mathrm{K} \mathrm{min}^{-1}\right)\end{array}$ & $T_{g}(\mathrm{~K})$ & $\Delta T_{g}(\mathrm{~K})$ & $\Delta c_{p}\left(\mathrm{~J} \mathrm{~K}^{-1} \mathrm{~mol}^{-1}\right)$ \\
\hline $\mathrm{H}_{2} \mathrm{O}$ ice IV [41] & 30 & $139.8 \pm 0.3$ & 6.4 & $1.2 \pm 0.2$ \\
$\mathrm{D}_{2} \mathrm{O}$ ice IV [41] & 30 & $146.2 \pm 0.8$ & & $0.5 \pm 0.1$ \\
$\mathrm{H}_{2} \mathrm{O}$ ice $\mathrm{V}^{\mathrm{a}}$ [35] & 30 & $130.0 \pm 0.1$ & & $1.7 \pm 0.2$ \\
$\mathrm{H}_{2} \mathrm{O}$ ice $\mathrm{V}$ (see Fig. 8) & 30 & $130.5 \pm 0.1$ & 7.1 & $2.3 \pm 0.3$ \\
$\mathrm{D}_{2} \mathrm{O}$ ice V (see Fig. 8) & 30 & $137.6 \pm 0.8$ & & $1.0 \pm 0.2$ \\
$\mathrm{H}_{2} \mathrm{O}$ ice $\mathrm{VI}^{\mathrm{b}}$ [29] & 10 & 132 & 6 & $\sim 2.5$ \\
$\mathrm{D}_{2} \mathrm{O}$ ice $\mathrm{VI}^{\mathrm{b}}$ [29] & 10 & 138 & & $\sim 1.5$ \\
$\mathrm{H}_{2} \mathrm{O}$ ice $\mathrm{VI}^{\mathrm{c}}$ [33] & 10 & $133.8 \pm 0.1$ & 4.3 & $\sim 1.8$ \\
$\mathrm{D}_{2} \mathrm{O}$ ice $\mathrm{VI}^{\mathrm{c}}$ [33] & 10 & $138.1 \pm 0.6$ & & $2.0 \pm 0.4$ \\
$\mathrm{H}_{2} \mathrm{O}$ ice VI (this work) & 10 & $128.1 \pm 0.6$ & 2.2 & $1.0 \pm 0.2$ \\
$\mathrm{D}_{2} \mathrm{O}$ ice VI (this work) & 10 & $130.3 \pm 0.4$ & & $1.9 \pm 0.2$ \\
$\mathrm{H}_{2} \mathrm{O}$ ice XII [35] & 30 & $130.0 \pm 1.0$ & & $1.5 \pm 0.2$ \\
$\mathrm{H}_{2} \mathrm{O}$ ice XII [41] & 30 & $131.4 \pm 0.8$ & $7.4 \pm 0.7$ & $1.4 \pm 0.2$ \\
$\mathrm{D}_{2} \mathrm{O}$ ice XII [41] & 30 & $138.1 \pm 0.4$ & & \\
\hline \hline
\end{tabular}

\footnotetext{
${ }^{a}$ We note that Salzmann et al. [28] have recorded an increase of heat capacity, $\Delta c_{p}=3.7 \mathrm{~J} \mathrm{~K}^{-1} \mathrm{~mol}^{-1}$, near the glass transition in ice V. This value does not correspond with those previously published [35] or measured in this work.

${ }^{\mathrm{b}}$ Heat capacity values extracted manually from Fig. 2 in Ref. [29].

${ }^{c}$ Heat capacity values extracted manually from Fig. 1 in Ref. [33].
}

orientational glass transition of ice V: Here, the $\mathrm{H}$ atoms become mobile, whereas the $\mathrm{O}$ atoms remain immobile [35]. We prepare ice $\mathrm{V}$ according to the literature protocol and measure its DSC scan both for pure $\mathrm{D}_{2} \mathrm{O}$ and $\mathrm{H}_{2} \mathrm{O}$. As evident from Fig. 9(b), the heat capacity change for the $\mathrm{D}_{2} \mathrm{O}$ sample $\left(1.0 \pm 0.2 \mathrm{~J} \mathrm{~K}^{-1} \mathrm{~mol}^{-1}\right)$ is much smaller than for the $\mathrm{H}_{2} \mathrm{O}$ variant $\left(2.3 \pm 0.3 \mathrm{~J} \mathrm{~K}^{-1} \mathrm{~mol}^{-1}\right)$. As summarized in Table I, similar results are seen for the isotope effect on the orientational glass transitions in ice IV and ice VI. In both cases, the $\Delta c_{p}$ is roughly halved if $\mathrm{D}_{2} \mathrm{O}$ is used instead of $\mathrm{H}_{2} \mathrm{O}$. The majority of these measurements show that an orientational glass transition reveals a significant $\mathrm{H} / \mathrm{D}$-isotope effect on $\Delta c_{p}$. The only exception is ice XII, for which substitution of $\mathrm{H}$ by $\mathrm{D}$ lowers $\Delta c_{p}$ only slightly. By contrast to the orientational glass transition for crystalline ices, there is no significant reduction of $\Delta c_{p}$ for the amorphous ices. Table II includes data for LDA, hyperquenched glassy water (HGW) from the literature, as well as the eHDA data from the current work. In all of these situations, there is barely any isotope effect on $\Delta c_{p}$ upon $\mathrm{H} / \mathrm{D}$ substitution. For $\mathrm{D}_{2} \mathrm{O} \mathrm{HGW}, \Delta c_{p}$ is even slightly larger than for $\mathrm{H}_{2} \mathrm{O} \mathrm{HGW}[51,52]$. This contrasts with the isotope effect on the volume, which was claimed to be normal for high-density liquid water but "anomalous" for low-density liquid water at low temperatures [53].

Thus, the isotope effect on the $\Delta c_{p}$ at the glass transition in amorphous ices is different from the one on the orientational glass transition in crystalline ices. Therefore, the atoms starting to be mobile near the glass transition cannot be the same when comparing crystalline and amorphous ices. We suggest that the huge isotope effect for the orientational glass transition is based on the fact that the mass of the mobile atoms is doubled by H/D substitution. By contrast, the mass of the mobile atoms is increased only by around $10 \%$ by $\mathrm{H}_{2} \mathrm{O} / \mathrm{D}_{2} \mathrm{O}$ substitution if the water molecule starts to translate at the glass transition, as is the case for a glass-to-liquid transition. As a consequence, only a very small isotope effect is expected for the unlocking of translational mobility at the glass transition, whereas a large isotope effect (reduction of $\Delta c_{p}$ by a factor of approximately 2) is seen for an orientational glass transition.

Thus, the data in Fig. 8(b) suggest that the nature of the glass transition in eHDA is a glass-to-liquid transition and not an orientational glass transition. This contrasts with conclusions by Shephard and Salzmann [33], who favor the latter scenario. Their conclusion is based on ${ }^{18} \mathrm{O}$-substitution experiments on eHDA and especially on their finding that $T_{g}$ (eHDA) does not shift upon exchanging naturally abundant oxygen by ${ }^{18} \mathrm{O}$. In our view, this observation does not compellingly rule out that translational motions accompany the glass transition. In fact, based on the following argument, we expect $T_{g}$ to remain unaffected upon use of ${ }^{18} \mathrm{O}$ samples, no matter which of the two scenarios is considered: Replacing the naturally abundant ${ }^{16} \mathrm{O}$ by ${ }^{18} \mathrm{O}$, the mass of the mobile atoms changes by only $10 \%$ if $\mathrm{O}$-atom motion is involved, and it does not change at all $(0 \%)$ if merely $\mathrm{H}$-atom motion is involved. At the melting transition of ice $I_{h}$, translational O-atom motion sets in. Nevertheless, the melting temperature of ${ }^{18} \mathrm{O}$-substituted hexagonal ice increases by only $0.1 \%$ compared to that of $\mathrm{H}_{2} \mathrm{O}$ ice of natural isotopic composition (273.43 vs 273.15 K) [54]. The observations by Shephard 
TABLE II. Onset glass transition temperatures $\left(T_{g}\right)$, difference in $T_{g}$ between $\mathrm{H}_{2} \mathrm{O}$ and $\mathrm{D}_{2} \mathrm{O}\left(\Delta T_{g}\right)$, and change in heat capacity $\left(\Delta c_{p}\right)$ at the glass transition for different $\mathrm{H}_{2} \mathrm{O}$ and $\mathrm{D}_{2} \mathrm{O}$ amorphous ices.

\begin{tabular}{lcccc}
\hline \hline & $\begin{array}{c}\text { Heating rate } \\
\left(\mathrm{K} \mathrm{min}^{-1}\right)\end{array}$ & $T_{g}(\mathrm{~K})$ & $\Delta T_{g}$ & $\Delta c_{p}\left(\mathrm{~J} \mathrm{~K}^{-1} \mathrm{~mol}^{-1}\right)$ \\
\hline $\mathrm{H}_{2} \mathrm{O}$ LDA [55] & 30 & 137 & 3 & 1.7 \\
$\mathrm{D}_{2} \mathrm{O}$ LDA [55] & 30 & 140 & & 1.5 \\
$\mathrm{H}_{2} \mathrm{O}$ HGW [52] & 30 & 136 & 1 & $1.6 \pm 0.1$ \\
$\mathrm{D}_{2} \mathrm{O}$ HGW [52] & 30 & 137 & & $1.8 \pm 0.2$ \\
$\mathrm{H}_{2} \mathrm{O}$ eHDA & b.07 [14] & 10 & 116 & 4.8 \\
$\mathrm{H}_{2} \mathrm{O}$ eHDA & (this work) & 50 & $125 \pm 0.9$ & $4.3 \pm 0.8$ \\
$\mathrm{D}_{2} \mathrm{O}$ eHDA \\
\hline \hline
\end{tabular}

${ }^{a}$ Other values from LDA or eHDA from the literature are not included in our table since they refer to other preparation methods. Specifically, $\mathrm{LDA}_{I}$ contains "nanocrystalline remnants," and eHDA decompressed to higher pressures, such as $0.3 \mathrm{GPa}$, cannot be considered a relaxed state [39]. The index for eHDA refers to the pressure in gigapascals to which the samples are decompressed.

${ }^{\mathrm{b}}$ Ruiz et al. [15] suggested $2.3 \pm 0.5 \mathrm{~J} \mathrm{~K}^{-1} \mathrm{~mol}^{-1}$ on the basis of the first plateau in the $c_{p}$ curve that is not observed in Fig. 7.

and Salzmann are consistent with a $T_{g}$ shift of only $0.1 \%$ [33]. Hence, we also regard them to be consistent with translational motion being activated at $T_{g}$ (eHDA).

A related remarkable difference concerns the isotope shift $\Delta T_{g}$ of the glass transition temperature. For the glassto-liquid transition of amorphous ices, this shift amounts to $1-3 \mathrm{~K}$ at $30 \mathrm{~K} \mathrm{~min}^{-1}$ (see Table II in this paper and Fig. 2 in Ref. [46]), while for orientational glass transitions in crystalline ices, it is $6-7 \mathrm{~K}$ at $30 \mathrm{~K} \mathrm{~min}^{-1}$ (see Table I). This difference with respect to $\Delta T_{g}$ can also be explained based on our previous argument that the reduced mass of the mobile atoms near the glass transition cannot be the same for crystalline and amorphous ices.

In absolute numbers, the heat capacity for noncrystalline $\mathrm{D}_{2} \mathrm{O}$ is higher than for $\mathrm{H}_{2} \mathrm{O}$. For instance, liquid water at ambient conditions has a heat capacity of $84.42 \mathrm{~J} \mathrm{~K}^{-1} \mathrm{~mol}^{-1}$ for $\mathrm{D}_{2} \mathrm{O}$ and $74.44 \mathrm{~J} \mathrm{~K}^{-1} \mathrm{~mol}^{-1}$ for $\mathrm{H}_{2} \mathrm{O}$ [56]. But why, in crystalline ices, is the heat capacity difference $\Delta c_{p}$ at $T_{g}$ smaller for $\mathrm{D}_{2} \mathrm{O}$ than it is for $\mathrm{H}_{2} \mathrm{O}$ ? We suggest that this can be understood on the basis of quantum tunneling. Since DSC measures merely relative changes in heat capacity, it is unknown how to offset the curves in Fig. 9(b) to reflect absolute heat capacities for the $\mathrm{D}_{2} \mathrm{O}$ and $\mathrm{H}_{2} \mathrm{O}$ samples. In Fig. 9(c), we offset the curves in a way so that the $\mathrm{D}_{2} \mathrm{O}$ phase above $T_{g}$ appears at a slightly higher $c_{p}$ than the $\mathrm{H}_{2} \mathrm{O}$ phase, just like it is the case for liquid water or hexagonal ice. Under these premises, it follows that the $\mathrm{H}_{2} \mathrm{O}$ phase has a much lower $c_{p}$ below $T_{g}$ than the $\mathrm{D}_{2} \mathrm{O}$ phase. This could be explained by quantum tunneling enhancing $\mathrm{H}$-atom motion much more than D-atom motion. Exactly, such a lowering of $c_{p}$ based on quantum tunneling in $\mathrm{H}_{2} \mathrm{O}$ ice was demonstrated theoretically by Vega et al. [57]. These authors concluded from their work that "the heat capacity is indeed one of the signatures of nuclear quantum effects in water." Thus, quantum tunneling in the low-temperature phase plays an important role in an orientational glass transition. However, we do not observe this kind of effect in amorphous ice, thereby ruling out that molecular reorientation is at the origin of the motions mobilized at $T_{g}$. With respect to the isotope effect on $\Delta c_{p}$, ice XII seems to be an exception among the crystalline ices. Currently, we are unable to rationalize why quantum tunneling would barely enhance the $\mathrm{H}$-atom mobility for ice XII, in contrast to the situation for ices IV, V, and VI. Our observation that $\Delta c_{p}$ in eHDA is only approximately $10 \%$ lower after $\mathrm{H} \rightarrow \mathrm{D}$ isotope substitution is, therefore, evidence that the nature of eHDA's $T_{g}$ involves not only $\mathrm{H}$-atom motion but also O-atom motion; i.e., it is a glassliquid transition rather than an orientational glass transition.

The published dielectric timescales also signal differences in the isotope effects of the crystalline versus those of the amorphous ices. While approaching the orientational glass transitions of ice $\mathrm{V}$ (and also that of ice XII) upon cooling, the ratio of the corresponding timescales $\tau(\mathrm{D}) / \tau(\mathrm{H})$ increases significantly $[24,45]$. On the other hand, any such increase near the glass transitions of the amorphous ices is only very weakly developed or even absent $[46,47]$.

To highlight these findings, in Fig. 10 we summarize the dielectric timescales of hydrogenated and deuterated ice $\mathrm{V}$ and those of eHDA together with lines highlighting their thermally activated behaviors. It is seen that the lines are parallel for eHDA $\mathrm{D}_{2} \mathrm{O}$ and eHDA $\mathrm{H}_{2} \mathrm{O}$ (corresponding to a temperature-independent ratio of timescales) but not parallel for ice $\mathrm{V} \mathrm{D}_{2} \mathrm{O}$ and ice $\mathrm{V}_{2} \mathrm{O}$. Towards lower temperatures, the dynamics in ice $\mathrm{V} \mathrm{H}_{2} \mathrm{O}$ slows down much less than it does in ice $\mathrm{V} \mathrm{D}_{2} \mathrm{O}$. More quantitatively, for ice $\mathrm{V}$ $\mathrm{H}_{2} \mathrm{O}$, the activation energy is $E=34 \mathrm{~kJ} / \mathrm{mol}$, and for ice $\mathrm{V}$ $\mathrm{D}_{2} \mathrm{O}$ it amounts to $51 \mathrm{~kJ} / \mathrm{mol}$ [24]. Hence, when lowering the temperature for ice $\mathrm{V}$, the timescale ratio increases from about 12 at $138 \mathrm{~K}$ to 70 at $125 \mathrm{~K}$. This observation is compatible with the notion that towards lower temperature, quantum tunneling enhances the dielectric dynamics of 


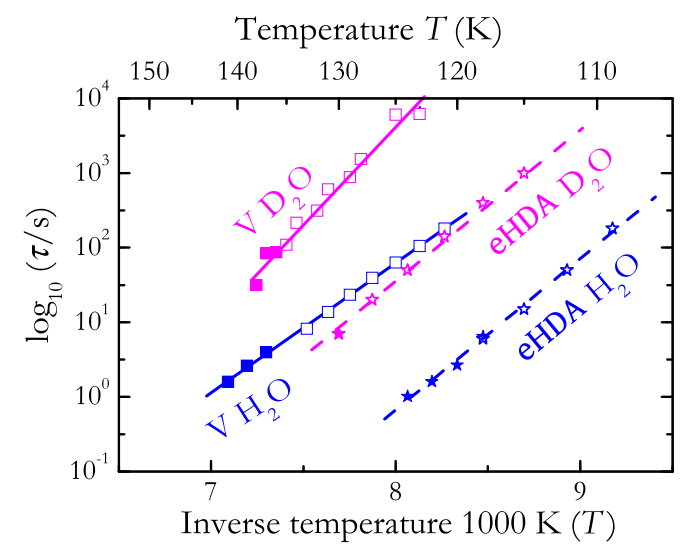

FIG. 10. Isotope effect on the dielectric timescales for ice V [24] and eHDA [47]. Similar to dielectric results for ice XII [45], the $\tau(\mathrm{D}) / \tau(\mathrm{H})$ ratio displays a significant temperature dependence also for ice V. Conversely, for eHDA the $\tau(\mathrm{D}) / \tau(\mathrm{H})$ ratio is constant.

ice $\mathrm{V} \mathrm{H}_{2} \mathrm{O}$ much more than that of ice $\mathrm{V}_{2} \mathrm{O}$. This is because only the H/D subnetworks are mobile above the orientational glass transition, which increases the tunneling probability by a factor of $1.41(\sqrt{2 / 1})$. For amorphous ices, however, a cooling-induced enhancement of timescales is not observed when comparing the isotopic species of eHDA: The dielectrically determined timescale ratio remains at approximately 50 between 135 and $115 \mathrm{~K}$ for eHDA. This isotope insensitivity implies that not only the $\mathrm{H} / \mathrm{D}$ subnetwork is mobile in eHDA above its $T_{g}$ but also the $\mathrm{O}$ network. If translational motion is operative, the tunneling probability does not significantly differ between $\mathrm{D}_{2} \mathrm{O}$ and $\mathrm{H}_{2} \mathrm{O}(\sqrt{20 / 18})$. This finding is in agreement with the conclusions drawn from the calorimetric data presented in Fig. 9 that the quantum effects are not strongly temperature dependent in eHDA but very much so in ice V.

\section{CONCLUSION}

In this work, we study the influence of dopants on amorphous ice, with a focus on eHDA samples. While $\mathrm{HCl}$ doping in crystalline ices is shown to enhance the dielectric dynamics by up to 5 orders of magnitude, none of the studied dopants enhances the dielectric dynamics of eHDA or LDA. Furthermore, $\mathrm{HCl}$ doping allows one to unlock the transition to the $\mathrm{H}$-ordered state in many crystalline ices. By contrast, in doped eHDA, neither transitions within the $\mathrm{O}$ network nor the $\mathrm{H}$ network are affected. Thus, $\mathrm{HCl}$ does not enhance the dynamics, just like all the other dopants tested in this work. Additionally, for none of the dopants could we observe tendencies towards local hydrogen ordering at low temperatures. These findings are counter to a scenario which assumes a crystallinelike nature of eHDA governed by point-defect dynamics. Obviously, the nature of the amorphous structure does not allow dopants to introduce relevant point defects that would speed up the $\mathrm{H}$ dynamics. Based on the dielectric results, similar conclusions can additionally be drawn for LDA. Also, here the (missing) doping-induced enhancement of the $\mathrm{H}$-atom dynamics clearly differs from the findings for crystalline ices, such as ice I, ice V, and ice XII. Let us also draw attention to recent classical molecular dynamics simulations, which demonstrate that polydispersed ice domains are lacking in both LDA and eHDA [58].

From the calorimetry results in Fig. 7, it is also evident that none of the dopants influences the calorimetric glass transition phenomenology; i.e., $\Delta c_{p}$ and $T_{g}$ remain the same for doped and undoped samples. That is to say, we have no indications of a scenario that involves the (un)freezing of point defects in the sense suggested by Fisher and Devlin [34] regarding the origin of the glass transition in eHDA or LDA. The kind of motion that is (un)frozen instead at the glass transition is suggested from the present H/D-isotope substitution experiments. These experiments indicate that the heat capacity increase near $T_{g}$ is approximately halved for orientational $\mathrm{H}$-atom motion and accompanied by a shift in $T_{g}$ of 6-7 K for ices IV, V, and VI. For amorphous ices, however, we measure barely any $\mathrm{H} / \mathrm{D}$-isotope influence on $\Delta c_{p}$ and a $T_{g}$ shift of only $1-3 \mathrm{~K}$. This isotope effect is very similar to the one observed at the melting transition of ice, where translational motion sets in. Consistent with the findings from dopant studies, also the isotope substitution studies can be explained only on the basis of nonorientational motions of water molecules, which are at the origin of the insensitivity of $\Delta c_{p}$ to isotope exchange. In order to learn about the mass of the mobile atoms at water's second glass transition, we study the dielectric relaxation timescale ratio $\tau(\mathrm{D}) / \tau(\mathrm{H})$ and activation energies in Fig. 10. This ratio is found to be insensitive to temperature changes for amorphous ices but not for ice $V$. The activation energy increases upon deuteration for ice $\mathrm{V}$ but not so for eHDA. The change in activation energy in ice $\mathrm{V}$ is explained by quantum tunneling strongly increasing towards low temperatures in the $\mathrm{H}$ subnetwork but barely in the $\mathrm{D}$ subnetwork. The insensitivity in amorphous ices is explained by whole water molecules being mobile rather than just the H/D subnetworks, which is again suggestive of translational molecular motion becoming possible above water's second glass transition. These findings are consistent with the idea of a glass-to-liquid transition in eHDA (at $116 \mathrm{~K}$ ) but not with that of a glass-to-glass transition. This conclusion is in accord with a recent analysis of x-ray speckle patterns suggesting diffusive dynamics in eHDA to occur prior to its polyamorphic transition [59]. Thus, the contested nature of water's second glass transition is clarified, with strong support for the existence of high-density liquid water above its $T_{g}$.

\section{ACKNOWLEDGMENTS}

Support for this project by the Deutsche Forschungsgemeinschaft under Grant No. BO1301/15-1 and the 
Austrian Science Fund (FWF) bilateral Project No. I1392 is gratefully acknowledged. V. F. L. is a recipient of a DOC fellowship of the Austrian Academy of Sciences ÖAW.

[1] O. Mishima, L. D. Calvert, and E. Whalley, An Apparently First-Order Transition between Two Amorphous Phases of Ice Induced by Pressure, Nature (London) 314, 76 (1985).

[2] O. Mishima, L. D. Calvert, and E. Whalley, Melting Ice I at $77 \mathrm{~K}$ and 10 kbar: A New Method of Making Amorphous Solids, Nature (London) 310, 393 (1984).

[3] E. Mayer, New Method for Vitrifying Water and Other Liquids by Rapid Cooling of Their Aerosols, J. Appl. Phys. 58, 663 (1985).

[4] I. Kohl, L. Bachmann, A. Hallbrucker, E. Mayer, and T. Loerting, Liquid-like Relaxation in Hyperquenched Water at $\leq 140$ K, Phys. Chem. Chem. Phys. 7, 3210 (2005).

[5] E. F. Burton and W. F. Oliver, X-Ray Diffraction Patterns of Ice, Nature (London) 135, 505 (1935).

[6] K. P. Stevenson, G. A. Kimmel, Z. Dohnálek, R. S. Smith, and B. D. Kay, Controlling the Morphology of Amorphous Solid Water, Science 283, 1505 (1999).

[7] R. J. Nelmes, J. S. Loveday, T. Strassle, C. L. Bull, M. Guthrie, G. Hamel, and S. Klotz, Annealed High-Density Amorphous Ice under Pressure, Nat. Phys. 2, 414 (2006).

[8] T. Loerting, C. Salzmann, I. Kohl, E. Mayer, and A. Hallbrucker, A Second Distinct Structural "State" of High-Density Amorphous Ice at $77 \mathrm{~K}$ and 1 bar, Phys. Chem. Chem. Phys. 3, 5355 (2001).

[9] J. Stern, M. Seidl, C. Gainaru, V. Fuentes-Landete, K. Amann-Winkel, P. H. Handle, K. W. Köster, H. Nelson, R. Böhmer, and T. Loerting, Experimental Evidence for Two Distinct Deeply Supercooled Liquid States of WaterResponse to "Comment on 'Water's Second Glass Transition,”, by G.P. Johari, Thermochim. Acta (2015), Thermochim. Acta 617, 200 (2015).

[10] P. H. Handle, M. Seidl, and T. Loerting, Relaxation Time of High-Density Amorphous Ice, Phys. Rev. Lett. 108, 225901 (2012).

[11] K. Winkel, E. Mayer, and T. Loerting, Equilibrated HighDensity Amorphous Ice and Its First-Order Transition to the Low-Density Form, J. Phys. Chem. B 115, 14141 (2011).

[12] Y. P. Handa, O. Mishima, and E. Whalley, High-Density Amorphous Ice. III. Thermal Properties, J. Chem. Phys. 84, 2766 (1986).

[13] M. Seidl, K. Amann-Winkel, P. H. Handle, G. Zifferer, and T. Loerting, From Parallel to Single Crystallization Kinetics in High-Density Amorphous Ice, Phys. Rev. B 88, 174105 (2013).

[14] K. Amann-Winkel, C. Gainaru, P. H. Handle, M. Seidl, H. Nelson, R. Böhmer, and T. Loerting, Water's Second Glass Transition, Proc. Natl. Acad. Sci. U.S.A. 110, 17720 (2013).

[15] G. N. Ruiz, K. Amann-Winkel, L. E. Bove, H. R. Corti, and T. Loerting, Calorimetric Study of Water's Two Glass Transitions in the Presence of $\mathrm{LiCl}$, Phys. Chem. Chem. Phys. 20, 6401 (2018).

[16] P. V. Hobbs, Ice Physics (Clarendon Press, Oxford, 1974).
[17] According to Ref. [18], the potassium ion presumably occupies an interstitial site of the lattice.

[18] V. F. Petrenko and R. W. Whitworth, Physics of Ice (Oxford University Press, New York, 1999).

[19] S. Kawada, Dielectric Dispersion and Phase Transition of KOH Doped Ice, J. Phys. Soc. Jpn. 32, 1442 (1972).

[20] Y. Tajima, T. Matsuo, and H. Suga, Calorimetric Study of Phase Transition in Hexagonal Ice Doped with Alkali Hydroxides, J. Phys. Chem. Solids. 45, 1135 (1984).

[21] T. Matsuo, Y. Tajima, and H. Suga, Calorimetric Study of a Phase Transition in $\mathrm{D}_{2} \mathrm{O}$ Ice Ih Doped with KOD: Ice XI, J. Phys. Chem. Solids 47, 165 (1986).

[22] C. G. Salzmann, P. G. Radaelli, A. Hallbrucker, E. Mayer, and J.L. Finney, The Preparation and Structures of Hydrogen Ordered Phases of Ice, Science 311, 1758 (2006).

[23] C. G. Salzmann, P. G. Radaelli, E. Mayer, and J. L. Finney, Ice XV: A New Thermodynamically Stable Phase of Ice, Phys. Rev. Lett. 103, 105701 (2009).

[24] K. W. Köster, A. Raidt, V. Fuentes-Landete, C. Gainaru, T. Loerting, and R. Böhmer, Doping-Enhanced Dipolar Dynamics in Ice V as a Precursor of Hydrogen Ordering in Ice XIII, Phys. Rev. B 94, 184306 (2016).

[25] K. W. Köster, V. Fuentes-Landete, A. Raidt, M. Seidl, C. Gainaru, T. Loerting, and R. Böhmer, Dynamics Enhanced by $\mathrm{HCl}$ Doping Triggers $60 \%$ Pauling Entropy Release at the Ice XII-XIV Transition, Nat. Commun. 6, 7349 (2015).

[26] K. W. Köster, T. Klocke, F. Wieland, and R. Böhmer, Interplay of Defect Doping and Bernal-Fowler Rules: A Simulation Study of the Dynamics on Ice Lattices, Phys. Rev. B 96, 134301 (2017).

[27] A. Rosu-Finsen and C. G. Salzmann, Benchmarking Acid and Base Dopants with Respect to Enabling the Ice V to XIII and Ice VI to XV Hydrogen-Ordering Phase Transitions, J. Chem. Phys. 148, 244507 (2018).

[28] C. G. Salzmann, P. G. Radaelli, J. L. Finney, and E. Mayer, A Calorimetric Study on the Low Temperature Dynamics of Doped Ice V and Its Reversible Phase Transition to Hydrogen Ordered Ice XIII, Phys. Chem. Chem. Phys. 10, 6313 (2008).

[29] J. J. Shephard and C. G. Salzmann, The Complex Kinetics of the Ice VI to Ice XV Hydrogen Ordering Phase Transition, Chem. Phys. Lett. 637, 63 (2015).

[30] A. I. Kolesnikov, V. V. Sinitsyn, E. G. Ponyatovsky, I. Natkaniec, and L.S. Smirnov, Similarity of Vibrational Spectra of High-Density Amorphous Ice and High-Pressure Phase Ice VI, Physica Amsterdam) 213B, 474 (1995).

[31] A. M. Saitta, T. Strässle, and S. Klotz, Temperature-Induced Topological Differentiation of the Two High-Density Amorphous Ices, Europhys. Lett. 74, 445 (2006).

[32] J. J. Shephard, S. Ling, G. C. Sosso, A. Michaelides, B. Slater, and C. G. Salzmann, Is High-Density Amorphous Ice Simply a "Derailed" State along the Ice I to Ice IV Pathway?, J. Phys. Chem. Lett. 8, 1645 (2017).

[33] J. J. Shephard and C. G. Salzmann, Molecular Reorientation Dynamics Govern the Glass Transitions of the Amorphous Ices, J. Phys. Chem. Lett. 7, 2281 (2016).

[34] M. Fisher and J. P. Devlin, Defect Activity in Amorphous Ice from Isotopic Exchange Data: Insight into the Glass Transition, J. Phys. Chem. 99, 11584 (1995). 
[35] C. G. Salzmann, I. Kohl, T. Loerting, E. Mayer, and A. Hallbrucker, The Low-Temperature Dynamics of Recovered Ice XII as Studied by Differential Scanning Calorimetry: A Comparison with Ice V, Phys. Chem. Chem. Phys. 5, 3507 (2003).

[36] K. Amann-Winkel, R. Böhmer, F. Fujara, C. Gainaru, B. Geil, and T. Loerting, Colloquium: Water's Controversial Glass Transitions, Rev. Mod. Phys. 88, 011002 (2016).

[37] O. Andersson and A. Inaba, Dielectric Properties of HighDensity Amorphous Ice under Pressure, Phys. Rev. B 74, 184201 (2006).

[38] G. P. Johari, A. Hallbrucker, and E. Mayer, Isotope and Impurity Effects on the Glass Transition and Crystallization of Pressure-Amorphized Hexagonal and Cubic Ice, J. Chem. Phys. 95, 6849 (1991).

[39] K. Winkel, M. S. Elsaesser, E. Mayer, and T. Loerting, Water Polyamorphism: Reversibility and (Dis)continuity, J. Chem. Phys. 128, 044510 (2008).

[40] K. Winkel, M. Bauer, E. Mayer, M. Seidl, M. S. Elsaesser, and T. Loerting, Structural Transitions in Amorphous $\mathrm{H}_{2} \mathrm{O}$ and $\mathrm{D}_{2} \mathrm{O}$ : The Effect of Temperature, J. Phys. Condens. Matter 20, 494212 (2008).

[41] C. G. Salzmann, E. Mayer, and A. Hallbrucker, Thermal Properties of Metastable Ices IV and XII: Comparison, Isotope Effects and Relative Stabilities, Phys. Chem. Chem. Phys. 6, 1269 (2004).

[42] The minimum temperature is evaluated for the crystallization since the peak minimum is well defined, by contrast to the onset point, which is obscured by enthalpy relaxation prior to the transformation.

[43] I. Kirshenbaum, Physical Properties and Analysis of Heavy Water (McGraw-Hill, New York, 1951).

[44] M. Tyagi and S. S. N. Murthy, Dielectric Relaxation in Ice and Ice Clathrates and Its Connection to the LowTemperature Phase Transition Induced by Alkali Hydroxides as Dopants, J. Phys. Chem. A 106, 5072 (2002).

[45] V. Fuentes-Landete, K. W. Köster, R. Böhmer, and T. Loerting, Thermodynamic and Kinetic Isotope Effects on the Order-Disorder Transition of Ice XIV to Ice XII, Phys. Chem. Chem. Phys. 20, 21607 (2018).

[46] C. Gainaru et al., Anomalously Large Isotope Effect in the Glass Transition of Water, Proc. Natl. Acad. Sci. U.S.A. 111, 17402 (2014).

[47] S. Lemke et al., Relaxation Dynamics and Transformation Kinetics of Deeply Supercooled Water: Temperature,
Pressure, Doping, and Proton/Deuteron Isotope Effects, J. Chem. Phys. 147, 034506 (2017).

[48] J. L. Finney, A. Hallbrucker, I. Kohl, A. K. Soper, and D. T. Bowron, Structures of High and Low Density Amorphous Ice by Neutron Diffraction, Phys. Rev. Lett. 88, 225503 (2002).

[49] D. T. Bowron, J. L. Finney, A. Hallbrucker, I. Kohl, T. Loerting, E. Mayer, and A. K. Soper, The Local and Intermediate Range Structures of the Five Amorphous Ices at $80 \mathrm{~K}$ and Ambient Pressure: A Faber-Ziman and BhatiaThornton Analysis, J. Chem. Phys. 125, 194502 (2006).

[50] T. Loerting, K. Winkel, M. Seidl, M. Bauer, C. Mitterdorfer, P. H. Handle, C. G. Salzmann, E. Mayer, J. L. Finney, and D. T. Bowron, How Many Amorphous Ices Are There?, Phys. Chem. Chem. Phys. 13, 8783 (2011).

[51] The corresponding study considers the undershoot before the increase of heat capacity for the evaluation of $\Delta c_{p}$.

[52] G. P. Johari, A. Hallbrucker, and E. Mayer, Isotope Effect on the Glass Transition and Crystallization of Hyperquenched Glassy Water, J. Chem. Phys. 92, 6742 (1990).

[53] B. Pamuk, P. B. Allen, and M. V. Fernández-Serra, Insights into the Structure of Liquid Water from Nuclear Quantum Effects on the Density and Compressibility of Ice Polymorphs, J. Phys. Chem. B 122, 5694 (2018).

[54] F. Steckel and S. Szapiro, Physical Properties of Heavy Oxygen Eater. Part 1.-Density and Thermal Expansion, Trans. Faraday Soc. 59, 331 (1963).

[55] M. S. Elsaesser, K. Winkel, E. Mayer, and T. Loerting, Reversibility and Isotope Effect of the Calorimetric Glass $\rightarrow$ Liquid Transition of Low-Density Amorphous Ice, Phys. Chem. Chem. Phys. 12, 708 (2010).

[56] M. Nakamura, K. Tamura, and S. Murakami, Isotope Effects on Thermodynamic Properties: Mixtures of $x\left(\mathrm{D}_{2} \mathrm{O}\right.$ or $\left.\mathrm{H}_{2} \mathrm{O}\right)+(1-x) \mathrm{CH}_{3} \mathrm{CN}$ at $298.15 \mathrm{~K}$, Thermochim. Acta 253, 127 (1995).

[57] C. Vega, M. M. Conde, C. McBride, J. L. F. Abascal, E. G. Noya, R. Ramirez, and L. M. Sesé, Heat Capacity of Water: A Signature of Nuclear Quantum Effects, J. Chem. Phys. 132, 046101 (2010).

[58] F. Martelli, N. Giovambattista, S. Torquato, and R. Car, Searching for Crystal-Ice Domains in Amorphous Ices, Phys. Rev. Mater. 2, 075601 (2018).

[59] F. Perakis et al., Diffusive Dynamics During the High-toLow Density Transition in Amorphous Ice, Proc. Natl. Acad. Sci. U.S.A. 114, 8193 (2017). 